\title{
Deregulated Aiolos Expression in Common Variable Immunodeficiency
}

\author{
Katy Billot $^{1}$, Issam Arrouss ${ }^{1}$, Gisela Garcia-Alvarez ${ }^{2}$, Eric Oksenhendler ${ }^{3}$, Gael Mouillot ${ }^{1}$, \\ Patrice Debre ${ }^{1}$ and Angelita Rebollo, ${ }^{*}$
}

\author{
${ }^{1}$ Immunologie Cellulaire et Tissulaire, Hôpital Pitié-Salpêtrière/Université Pierre et Marie Curie/ Inserm U543, 83, Bd \\ de l'hôpital 75013 Paris, France \\ ${ }^{2}$ Groupe Myologie, INSERM U787 UPMC-Paris VI, Faculté de Médecine Pitié-Salpêtrière, 105 bd de l'Hôpital, 75634 \\ Paris Cedex 13, France \\ ${ }^{3}$ Département d'Immunologie Clinique, Hôpital Saint-Louis, 1 Avenue Claude Vellefaux, 75010 Paris, France and DE- \\ FI Study Group
}

\begin{abstract}
Common variable immunodeficiency (CVID), a frequent primary immunodeficiency disease, is characterized, by defects involving $\mathrm{T}$ and $\mathrm{B}$ cells. The Aiolos transcription factor plays an important role in the control of B cell differentiation and proliferation and is modulated through alternative splicing. In order to assess the role of Aiolos in this human pathology, we analyzed the Aiolos isoforms distribution and expression. We demonstrated that the major Aiolos isoform expressed in PBMC's of healthy donors and CVID patients is hAio1, whereas the rest of the Aiolos isoforms showed homogenous distribution. In addition, we detected three new Aiolos isoforms in CVID patients that were non-detectable in healthy donors. Furthermore, we quantified Aiolos expression in T and B cells from CVID patients, given that both cell populations are altered in this pathology. Our results show an up-regulation of Aiolos expression in $\mathrm{T}$ and $\mathrm{B}$ cells from CVID patients compared to healthy donors that was confirmed at the protein level. Finally, we show a different cellular distribution of Aiolos in CVID patients compared to that found in healthy donors.
\end{abstract}

Keywords: Common variable immunodeficiency, Aiolos, Transcription factor.

\section{INTRODUCTION}

Common variable immunodeficiency (CVID), a frequent primary immunodeficiency disease, includes a heterogeneous group of diseases characterized by low concentration of serum-immunoglobulin of all switched immunoglobulin isotypes, defective specific-antibody production and increased susceptibility to infections of the respiratory and gastrointestinal tracts with encapsulated bacteria. Subgroups of patients have increased risks of some of the following conditions including splenomegaly, granulomatous lesions, autoimmune disease and tumoral transformation [1]. The basic molecular immunological defect of this pathology is still unknown and probably results from the heterogeneity of the phenotypic features of the disease.

Since CVID patients often present with hypogammaglobulinemia, presumably due to a default in memory and plasma B cells, phenotypic analysis of their lymphocyte subpopulations has primarily focused on B cells [2-4]. Different classifications have been proposed, among then, the classification in four groups: NoB group, with less that $1 \%$ of CD19 positive cells; MB1, with a defect in switched B cell memory; MB0, with a defect in switched and non switched B cell memory and, finally, MB2 group, with a phenotype similar to healthy donors.

*Address correspondence to this author at the Hôpital Pitié-Salpêtrière, Inserm U543, 83, Bâtiment CERVI, 83, Bd de l'hôpital 75013 Paris, France ; Tel: 33-142177527; Fax: 33-142177490;

E-mail: rebollo@chups.jussieu.fr
Aiolos is a lymphoid transcription factor that plays an essential role during $\mathrm{B}$ cell proliferation and maturation. Within adult hematopoietic progenitors, Aiolos is not expressed in pluripotent stem cells but is detected at low levels in multipotent progenitors. It expression is up-regulated as these progenitors become restricted to $\mathrm{T}$ and $\mathrm{B}$ cell fates. In addition, development of $\mathrm{B}$ cell lymphomas was frequently seen among Aiolos-deficient mice [5,6].

We show an up-regulation of the Aiolos transcription factor in CVID patients as well as a different subcellular localization compared to healthy donors for the first time. This new information should assist in understanding the pathogenesis of CVID and improving therapeutic strategies.

\section{MATERIALS AND METHODOLOGY}

\section{Isolation of $T$ and $B$ Cells from Healthy Donors and CVID Patients}

Cryopreserved PBMC samples were obtained after informed consent from CVID patients from different hospitals in France, according to a protocol approved by the institutional ethic committee. The patients were not receiving any immunosuppressive drugs at the time of evaluation but were under immunoglobulin substitution therapy. Fresh blood from healthy donors was collected by the Etablissement Francais du Sang and PBMCs were frozen after density gradient isolation. Total RNA was extracted from PBMCs of CVI D and healthy donors. T cells were isolated using positive selection by anti-CD3 Dynal magnetic beads. B cells 
were isolated using Dynal negative isolation kit (Invitrogen). The purity of the isolated $\mathrm{T}$ and $\mathrm{B}$ cells reached $98 \%$.

\section{RT-PCR}

Total RNA extraction was done using Qiagen RNeasy kit according to manufacturers' instructions. RNA $(1 \mu \mathrm{g})$ was reverse transcribed and amplified by Titanium one step RTPCR (Clontech). Aiolos forward, 5'-GGCAGCGACATGGA AG-3', Aiolos reverse 5'-TAGCTGATGGCGTTATTGATG G-3'. We used EF1 as internal control with the following primer sequences. EF1 forward, 5'-AAGAATGTGTCTGTC AAGGATGTTC-3', EF1 reverse 5'-GCCTGGATGGTTCA GGATAA-3'. The RT-PCR products were resolved by agarose gel electrophoresis and visualized by ethidium bromide staining.

\section{RT-(q)PCR Analysis}

RNA was reversed transcribed and amplified by superscript VILO cDNA synthesis kit (Invitrogen). Quantitative PCR was carried out on an ABI PRISM 7300 detection system. We used pre-developed TaqMan gene expression assay (Applied Biosystems) for quantitative detection of human Aiolos (Hs00232635 m1) and the absolute qPCR Rox mix (Abgene) according to the manufacturers' instructions. Data from triplicates are expressed as normalized expression by using the $2^{-\Delta \Delta \mathrm{Ct}}$ calculation method and the Abelson gene (Hs00245445_m1) as a reference gene.

\section{Western Blot}

Western blot was carried out as previously described [7].

\section{Peptide Synthesis and Antibody Production}

A specific peptide of the Aiolos amino acid sequence 368-384 was synthesized on an automated multiple peptide synthesizer (AMS422, Abimed, Langefeld, Germany) using the solid phase procedure and standard Fmoc chemistry. Purity and peptide composition were confirmed in reversephase HPLC and by amino acid analysis in a Beckman 6300 amino acid analyser. The peptide, glutaraldehyde-coupled to KLH via $\mathrm{N}$-terminal lysine, was injected into outbreed New Zeeland rabbits. Serum was collected 7-10 days after the last injection.

\section{Immunofluorescence and Confocal Analysis}

Immunofluorescence and confocal analysis was carried out as previously described [8]. Briefly, PBMC from CVID patients or healthy donors were fixed with $1 \%$ paraformaldehyde for $5 \mathrm{~min}$, permeabilized and then incubated with polyclonal anti-Aiolos antibody for $2 \mathrm{~h}$ in PBS/BSA at room temperature. FITC-secondary antibody was added and incubated for $1 \mathrm{~h}$ at room temperature. After several washing steps, samples were mounted and analyzed. In each experiment, we analyzed six CVID patients and seven healthy donors. The anti-Aiolos antibody has been previously described [9]. The anti-Bad antibody was from Abcam (Cambridge, UK).

\section{RESULTS AND DISCUSSION}

\section{Characterization of Aiolos Isoforms in CVID}

Given that the Aiolos transcription factor is involved in B cell maturation and that $\mathrm{B}$ cells are affected in CVID, we decided to quantitatively analyze Aiolos expression. We ana- lyzed the expression pattern of Aiolos by PCR in PBMCs of individuals diagnosed with CVID (4 MB0, 7 MB1, 4 MB2 and $4 \mathrm{NoB}$ ). Table 1 summarizes the phenotypic analysis of $\mathrm{B}$ and $\mathrm{T}$ cells from 19 CVID patients, as well as healthy donors. Table 2 shows the clinical data of CVID patients and healthy donors. PCR analysis of healthy donors reveals the presence of 6 Aiolos isoforms (Fig. 1A): hAio1, hAio2, hAio $3 / 4$, hAio5 and hAio-del $(4,5,6)$ [10-12]. Long isoforms, with at least three zinc fingers, can bind efficiently to DNA, while shorter versions behave as dominant negative isoforms upon heterodimerization. These short isoforms are normally expressed at low levels, compatible with their potential regulatory role on the activity of the predominant isoform. No major differences were observed in the distribution of isoforms between healthy donors and CVID samples. Interestingly, some Aiolos isoforms were differentially expressed in CVID patients and not detected in healthy donors (Fig. 1A). The isoforms differentially expressed in CVID patients might correspond to the isoforms described by Caballero et al. [13] in cell lines derived from B and T lymphoid malignancies. The new isoform expression pattern described by Caballero et al. [13] was not associated with particular cell or sample types. The new isoforms were also observed in B cells from individuals diagnosed with systemic lupus erythematosus and rheumatoid arthritis, two common autoimmune diseases. Aio1 was the predominant isoform detected in this work [13], similarly to our results.

In our CVID samples, the expression of the isoforms not detected in healthy donors is not associated with any particular group of patients. On the other hand, hAiol was the isoform with the most variability among patient groups (Fig. 1A). A similar up-regulation of hAiol was observed in cells from chronic lymphocytic leukaemia patients [11] as well as in the cases described by Caballero et al. [13].

Our results did not reveal any significant over-expression of Aiolos dominant negative isoforms in CVID patients compared to healthy donors. The imbalance observed between dominant negative and wild type isoforms might be involved in the pathogenesis of CVID by disturbing Aiolos subcellular localization and its association with HDACcontaining complexes [13].

We further quantify Aiolos expression in T and B cells of CVID, given that both populations seem to be altered in this human pathology. Aiolos expression was analyzed by a-PCR in $\mathrm{T}$ and $\mathrm{B}$ cells of CVID patients and healthy donors and Aiolos expression levels are compared among the four phenotypic groups (Fig. 1B). T cells from MB1 and MB2 patient groups show strong up-regulation of Aiolos expression compared to healthy donors. $T$ cells from patients within the MB0 group show a moderate up-regulation of Aiolos expression and finally, the NoB group shows a slightly higher Aiolos expression than healthy donors (Fig. 1B). Regarding the expression of Aiolos in B cells from CVID patients, we also observed a strong up-regulation of Aiolos, except in patients of the NoB group that have non-significant levels of B cells (Fig. 1B). It is interesting to notice that Aiolos expression is upregulated in both $\mathrm{T}$ and $\mathrm{B}$ cells of CVID patients (Fig. 1C), suggesting an alteration in both cell populations. Taken together, these results show that $\mathrm{T}$ and $\mathrm{B}$ cells express higher total levels of Aiolos in CVID patients compared to healthy donors. Our results show a quantitative and qualitative 
Table 1. Characteristics of the Four Groups of Patients and Healthy Donors Used in this Study

\begin{tabular}{|c|c|c|c|c|c|}
\hline CVID Patients & Sex & Age & Group & Years Since Onset & Years Since Diagnosis \\
\hline $\mathrm{P} 1$ & $\mathrm{~F}$ & 75 & MB0 & 64 & 17 \\
\hline $\mathrm{P} 2$ & $\mathrm{~F}$ & 21 & MB0 & 21 & 21 \\
\hline P3 & $\mathrm{M}$ & 36 & MB0 & 31 & 10 \\
\hline P4 & M & 28 & MB0 & 17 & 1 \\
\hline P5 & $\mathrm{F}$ & 25 & MB1 & 7 & 5 \\
\hline P6 & $\mathrm{F}$ & 50 & MB1 & 4 & 4 \\
\hline P7 & $\mathrm{F}$ & 49 & MB1 & 19 & 19 \\
\hline P8 & $\mathrm{F}$ & 36 & MB1 & 35 & 0 \\
\hline P9 & $\mathrm{F}$ & 55 & MB1 & 7 & 2 \\
\hline $\mathrm{P} 10$ & $\mathrm{~F}$ & 67 & MB1 & 16 & 3 \\
\hline P11 & $\mathrm{F}$ & 54 & MB1 & 24 & 24 \\
\hline $\mathrm{P} 12$ & $\mathrm{M}$ & 56 & MB2 & 4 & 0 \\
\hline P13 & $\mathrm{M}$ & 28 & MB2 & 11 & 0 \\
\hline P14 & $\mathrm{F}$ & 33 & MB2 & 6 & 1 \\
\hline P15 & $\mathrm{F}$ & 19 & MB2 & 1 & 0 \\
\hline P16 & $\mathrm{M}$ & 41 & NoB & 41 & 38 \\
\hline P17 & $\mathrm{F}$ & 33 & NoB & 3 & 0 \\
\hline P18 & $\mathrm{F}$ & 62 & NoB & 58 & 26 \\
\hline P19 & $\mathrm{F}$ & 16 & NoB & 16 & 5 \\
\hline
\end{tabular}

\begin{tabular}{|c|c|c|}
\hline HD & Sex & Age \\
\hline \hline HD1 & F & 50 \\
\hline HD2 & F & 48 \\
\hline HD3 & M & 53 \\
\hline HD4 & F & 24 \\
\hline HD5 & F & 50 \\
\hline HD6 & M & 19 \\
\hline HD7 & F & 38 \\
\hline HD8 & F & 23 \\
\hline HD9 & M & 40 \\
\hline
\end{tabular}

anomaly concerning Aiolos expression, in contrast to chronic lymphocytic leukaemia (CLL), which shows only a quantitative anomaly in Aiolos expression.

\section{Aiolos is Overexpressed in CVID}

The increase in Aiolos mRNA expression in CVID patients was also confirmed at the protein level, as shown in Fig. (2A). All patients show an up-regulation of Aiolos expression at the protein level, compared to healthy donors. Fig. (2B) shows the densitometric analysis of the Aiolos expression shown in Fig (2A). Similarly, Aiolos up-regulation has been observed in patients from CLL [11], but the consequences of this up-regulation are not yet known. Aiolos has been implicated in the control of Bcl-2 expression in T cells [9] but its implication in B cells has not been demonstrated
[14]. In our patients, Bcl-2 expression seems independent of Aiolos levels (data not shown). The susceptibility of $\mathrm{T}$ cells to undergo apoptosis is controlled by the Bcl-2 family proteins [15]. Over-expression of $\mathrm{Bcl}-2$ and $\mathrm{Bcl}-\mathrm{x}_{\mathrm{L}}$ enhances survival of $\mathrm{T}$ cells that are induced to undergo apoptosis. However, Di Renzo and co-workers [16] did not find any difference in Bcl-2 and Bcl- $\mathrm{x}_{\mathrm{L}}$ expression in CD4 or CD8 T cells between CVID patients and normal donors, in support of our observation in the present study.

Aiolos also appears to inhibit the threshold of BCR activation by modulating tyrosine kinase phosphorylation and calcium release to the cytoplasm $[5,14]$. A slight imbalance between dominant negative and anti-oncogenic Aiolos isoforms might be involved in the pathogenesis of CVID by 
Table 2. Phenotype of the Four Groups of Patients and Healthy Donors Used in this Study

\begin{tabular}{|c|c|c|c|c|c|c|c|c|c|c|c|}
\hline \multirow{3}{*}{$\begin{array}{c}\text { CVID } \\
\text { Patients }\end{array}$} & \multirow{3}{*}{ Group } & \multicolumn{6}{|c|}{ B Cells } & \multicolumn{4}{|c|}{ T Cells } \\
\hline & & \multicolumn{2}{|c|}{$\mathrm{CD}{ }^{+}$} & \multirow{2}{*}{$\frac{\mathrm{IgD}^{+} / \mathrm{CD}^{-} 7^{-}}{\%}$} & \multirow{2}{*}{$\frac{\mathrm{IgD}^{+} / \mathrm{CD}^{2} 7^{+}}{\%}$} & \multirow{2}{*}{$\frac{\mathrm{IgD}^{-} / \mathrm{CD}^{2} 7^{+}}{\%}$} & \multirow{2}{*}{$\begin{array}{c}\mathrm{CD}^{2} / \mathrm{CD}^{\circ}{ }^{+} \\
\%\end{array}$} & \multicolumn{2}{|c|}{$\mathrm{CD3}^{+}$} & \multirow{2}{*}{$\frac{\mathrm{CD4}^{+}}{\%}$} & \multirow{2}{*}{$\begin{array}{c}\mathrm{CD8}^{+} \\
\%\end{array}$} \\
\hline & & $\%$ & $/ \mathbf{m m}^{3}$ & & & & & $\%$ & $/ \mathbf{m m}^{3}$ & & \\
\hline $\mathrm{P} 1$ & MB0 & 19,6 & 384 & 94 & 6 & 1 & 7 & 56 & 1098 & 23 & 31 \\
\hline $\mathrm{P} 2$ & MB0 & 14,3 & 201 & 97 & 1 & 1 & 1 & 72 & 1014 & 33 & 32 \\
\hline P3 & MB0 & 12 & 227 & 98 & 1 & 0 & 6 & 75 & 1421 & 27 & 46 \\
\hline P4 & MB0 & 13,8 & 103 & 95 & 2 & 1 & 3 & 75 & 560 & 37 & 25 \\
\hline P5 & MB1 & 16,9 & 253 & 81 & 18 & 0 & 38 & 70 & 1048 & 60 & 7 \\
\hline P6 & MB1 & 17 & 279 & 63 & 30 & 5 & 9 & 77 & 1264 & 38 & 39 \\
\hline P7 & MB1 & 16,8 & 164 & 52 & 46 & 2 & 48 & 61 & 597 & 41 & 17 \\
\hline P8 & MB1 & 18,3 & 299 & 65 & 32 & 2 & 5 & 68 & 1111 & 29 & 36 \\
\hline P9 & MB1 & 14,6 & 183 & 69 & 28 & 1 & 15 & 68 & 851 & 48 & 16 \\
\hline P10 & MB1 & 14,1 & 204 & 84 & 13 & 2 & 17 & 71 & 1026 & 53 & 16 \\
\hline P11 & MB1 & 17,5 & 277 & 81 & 17 & 1 & 2 & 67 & 1061 & 46 & 17 \\
\hline P12 & MB2 & 7,2 & 120 & 65 & 13 & 8 & 3 & 81 & 1354 & 44 & 21 \\
\hline P13 & MB2 & 5,8 & 129 & 60 & 13 & 22 & 2 & 81 & 1805 & 43 & 33 \\
\hline P14 & MB2 & 9,7 & 126 & 63 & 22 & 11 & 3 & 77 & 999 & 52 & 24 \\
\hline P15 & MB2 & 10,6 & 146 & 61 & 26 & 9 & 5 & 77 & 1060 & 41 & 21 \\
\hline P16 & NoB & 0 & & & & & & 90 & 1522 & 61 & 27 \\
\hline P17 & NoB & 0,8 & & & & & & 88 & 1740 & 33 & 45 \\
\hline P18 & NoB & 0,5 & & & & & & 85 & 1097 & 42 & 22 \\
\hline P19 & NoB & 0 & & & & & & 78 & 2058 & 39 & 29 \\
\hline
\end{tabular}

\begin{tabular}{|c|c|c|c|c|c|c|c|c|c|c|c|}
\hline \multirow{3}{*}{ HD $(n=50)$} & median & 11 & 202 & 67 & 14,5 & 14,5 & 2 & 74 & 1418 & 45 & 25 \\
\hline & $\min$ & 57 & 85 & 34 & 6 & 4 & 0,4 & 61 & 542 & 34 & 15 \\
\hline & $\max$ & 20,8 & 623 & 87 & 49 & 40 & 7 & 85 & 2598 & 63 & 39 \\
\hline
\end{tabular}

disturbing Aiolos sub-cellular localization and its association with HDAC-containing complexes [17].

Based on our results, we can't conclude whether Aiolos over-expression is the cause or the consequence of the CVID pathology. We do not exclude the possibility that the mechanisms involved in Aiolos over-expression might involve mRNA half-life stabilization or even histone modifications, since the parameters implicated in the regulation of Aiolos are largely unknown. We have shown that DNA methylation of Aiolos promoter directs Aiolos silencing and chromatin status in tumour cell lines while expression of Aiolos in primary cells is mainly regulated by histone modifications [7, 11]. The up-regulation of Aiolos in CLL seems independent of euchromatin markers modification throughout the Aiolos promoter [11]. In addition, it has also been shown that Ikaros and NF- $\kappa \mathrm{B}$ transcription factors are critical for Aiolos promoter activity in vitro and in vivo [7].

It is well known that the regulation of protein localization can be used to control cell signalling pathways, therefore, we further analyzed the subcellular localization of Aiolos in PBMC by confocal microscopy (Fig. 2C). We observed that cells from healthy donors show lower Aiolos labelling compared to cells from CVID patients. Interestingly, there is a clear difference in Aiolos localization between normal and disease cells. While healthy cells display condensed nuclear staining, CVID patients' cells display Aiolos staining in the nucleus and throughout the cytoplasm. Staining with an antiBad antibody display similar distribution in controls and CVID patients. To date, Pax5, SLP-65 and Ikaros [7, 18, 19] are the only transcription factors described that control Aiolos expression either directly or indirectly. Therefore, these proteins are likely candidates to observe for modifications of expression, localization and association with HDAC complexes in CVID patients [17].

For the first time, we demonstrate that misregulation of Aiolos protein localization is associated with an immunological pathology such as CVID, with corresponding increases in Aiolos message and protein levels. The exact causes and consequences of Aiolos up-regulation in CVID patients are yet to be determined. We furthermore suggest that Aiolos could be included in the molecular diagnosis of CVID together with btk, CD40L and SH2D1A [20]. 
(A)

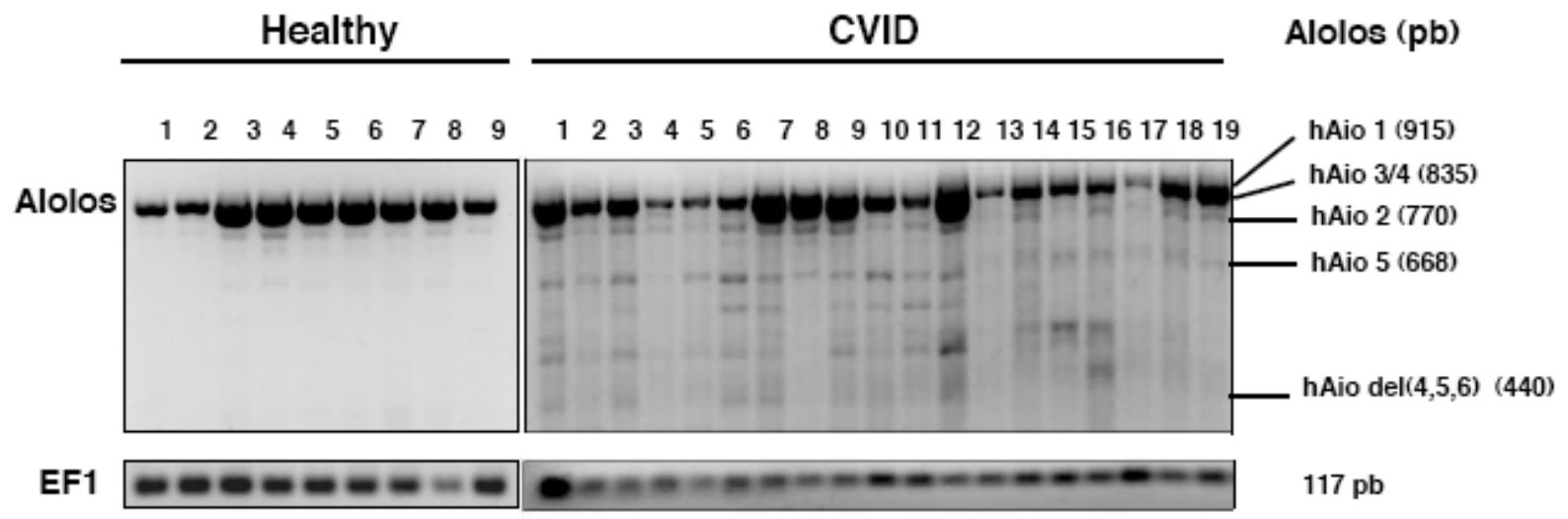

(B)

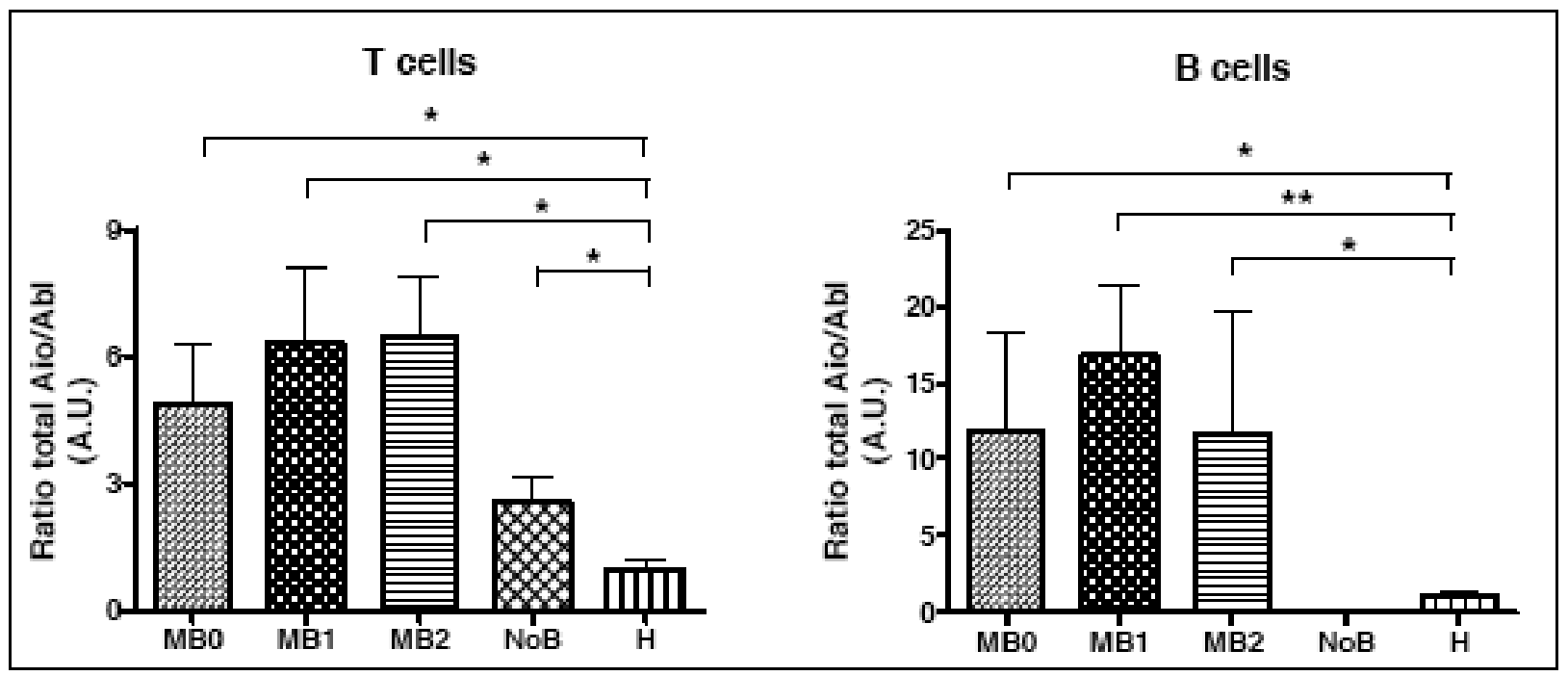

(C)

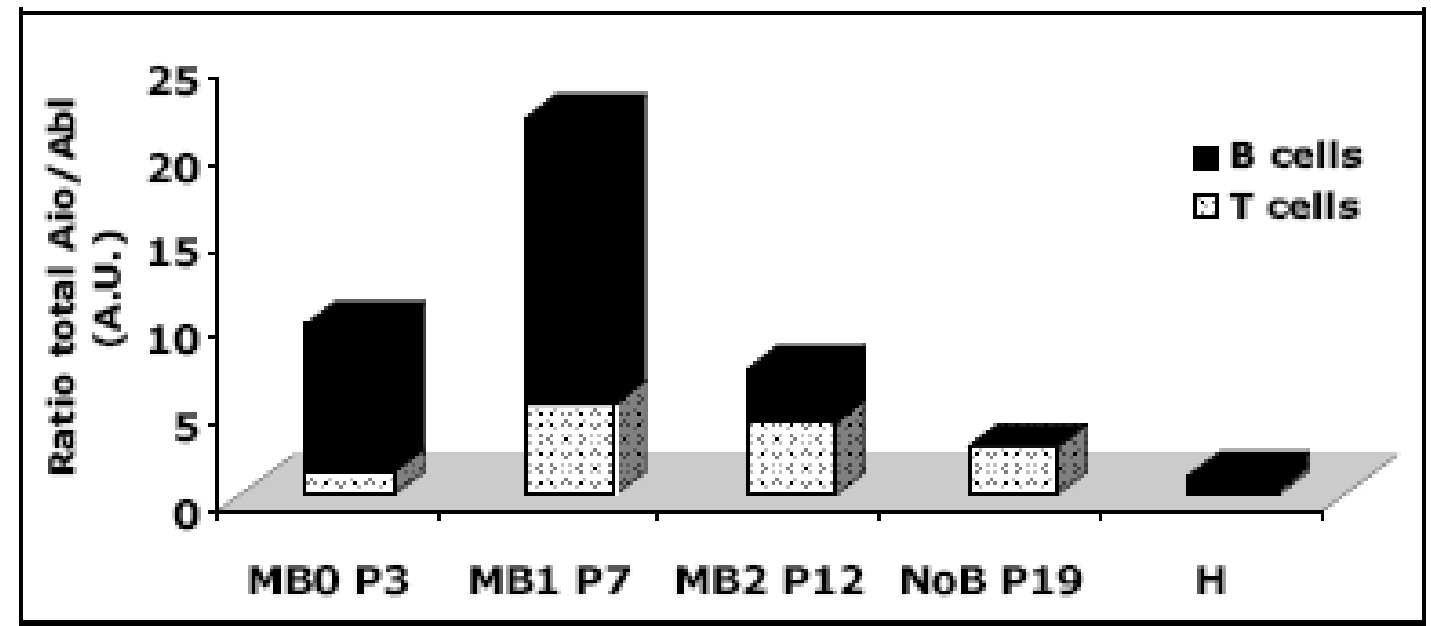

Fig. (1). Aiolos is up regulated in CVI patients at the mRNA level. (A) Representative results of Aiolos isoforms expression in healthy donor (H) and CVID patients after RT-PCR amplification of PBMC RNA. Elongation factor 1 (EF1) was used as internal control. The new Aiolos isoforms are marked by arrows and boxed. (B) Ratio of total Aiolos versus Abl expression in T and B cells after RT-q-PCR of the four groups of CVID patients. We analyzed four patients of each group and five healthy donors. (C) Ratio of total Aiolos versus Abl expression in $\mathrm{T}$ and B cells of patients 3, 6, 15 and 19. We used Prism4.0c (GraphPad Software, San Diego) for statistical analysis. Non parametric test with Mann-Whitney test was performed and statistical significances was set at $\mathrm{p} \leq 0.05$ with the following degrees: $* 0.01<\mathrm{p} \leq 0.05, * * 0.001$. Abl, kinase Abl used as internal control; A.U, arbitrary units. 
(A)

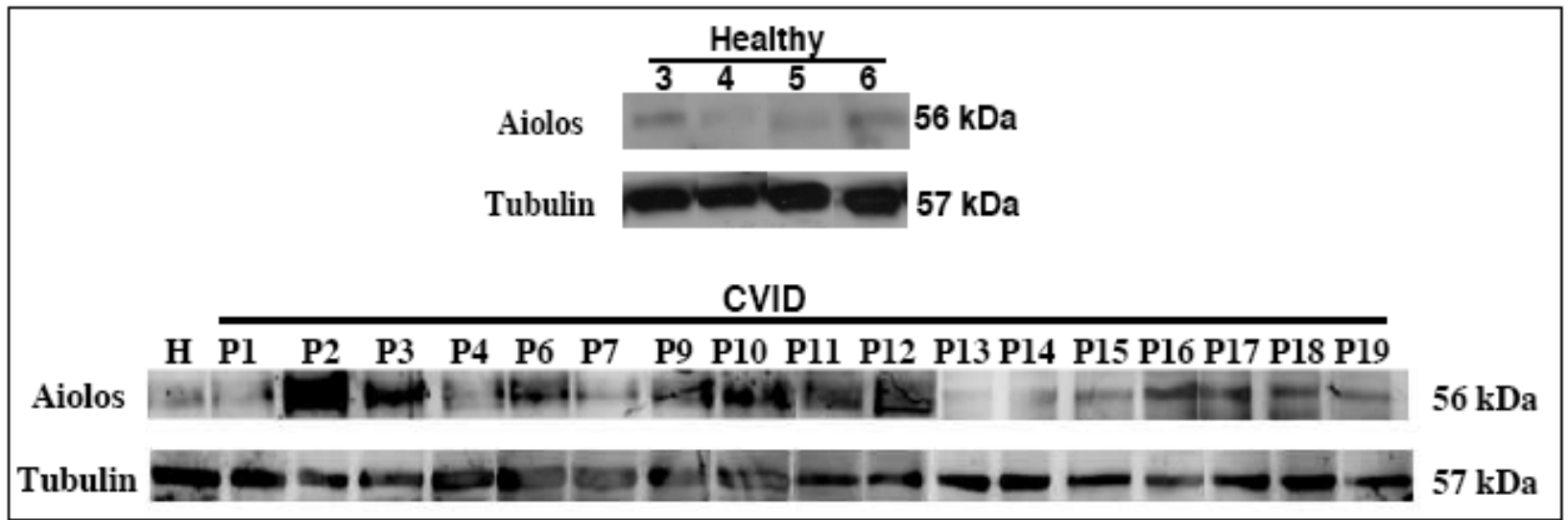

(B)

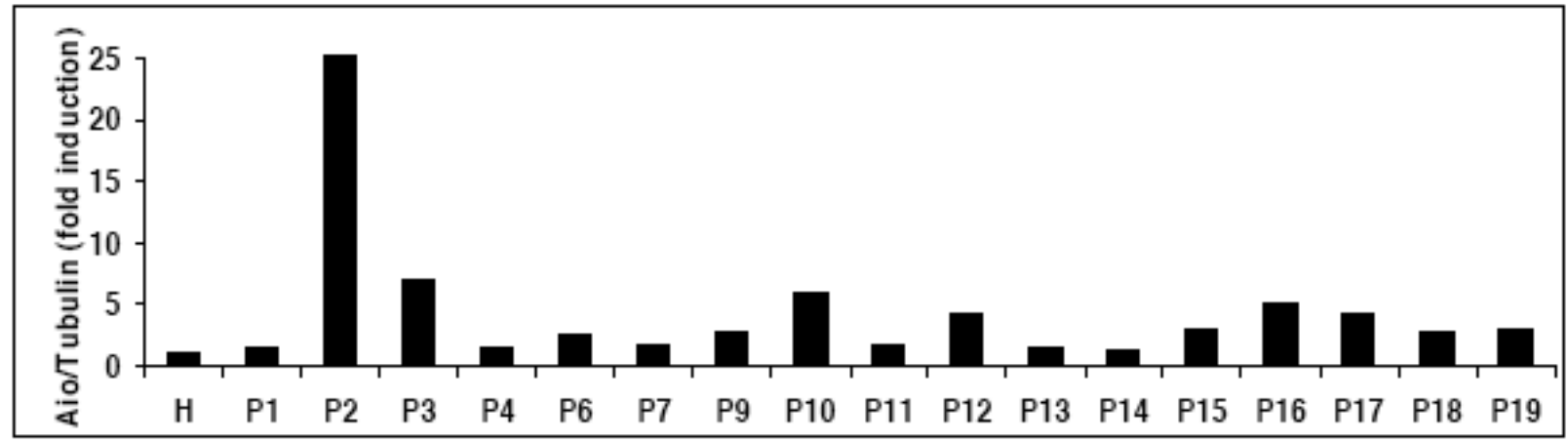

(C)

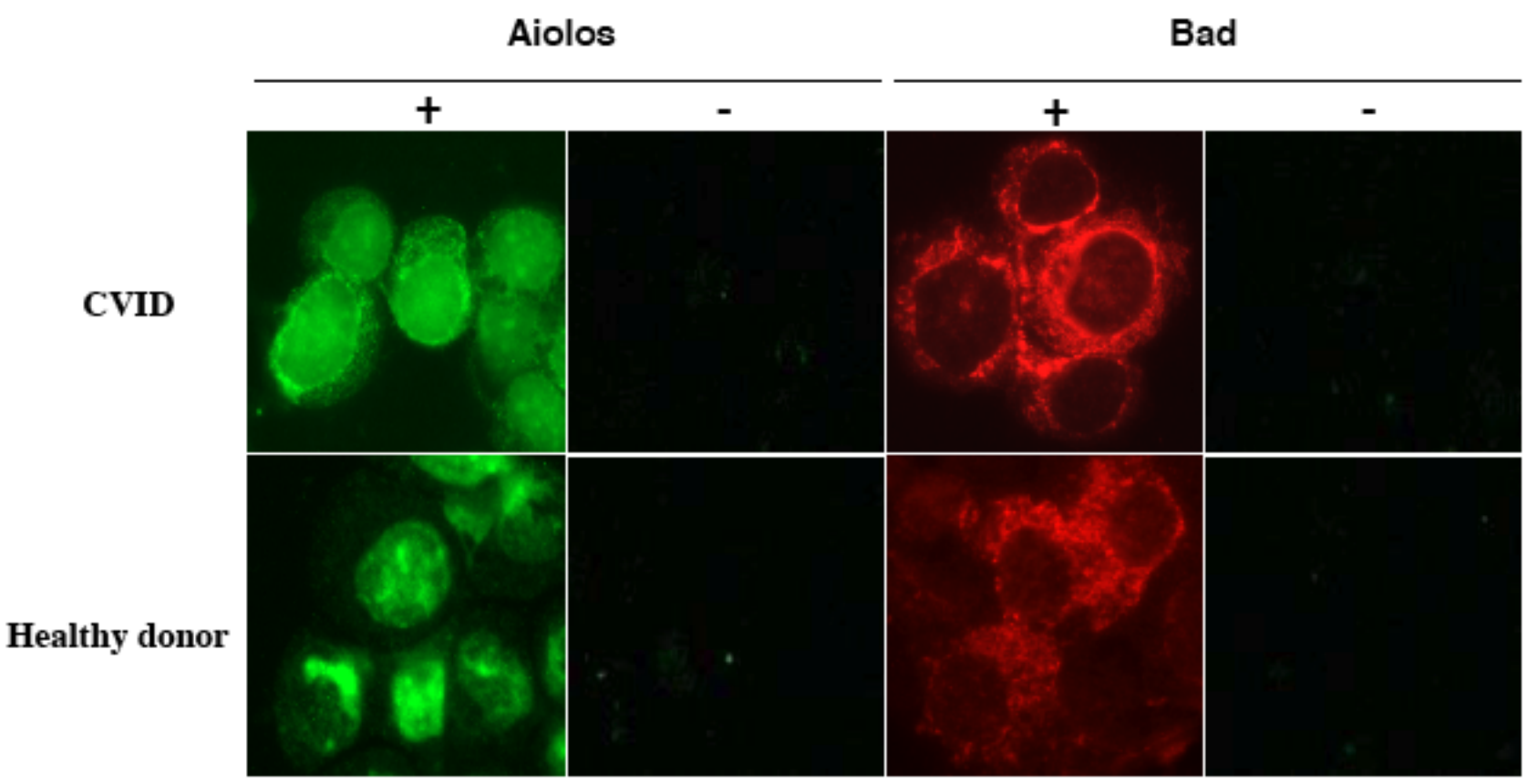

Fig. (2). Aiolos is up regulated in CVID patients at the protein level in PBMC cells. (A) Western blot analysis of Aiolos expression in healthy donors and CVID patients. Tubulin expression was used as internal control. Molecular weight of the proteins is shown. (B) Densitometric analysis of results on Fig. (2A). Similar results were obtained in three independent experiments. (C) Localization of Aiolos in healthy donors and CVID patients. Samples were stained with anti-Aiolos (rabbit polyclonal Ab), anti-Bad antibody (purified polyclonal Ab) or a pre-immune rabbit serum followed by a fluorescence secondary antibody (anti-rabbit FITC or Cy3) and analyzed by confocal microscopy. Similar results were obtained in three independent experiments. In each experiment, we analyzed six CVID patients randomly selected (one from MB0 group, two from MB1 group, two from MB2 group and one from NoB group) and seven healthy donors. 


\section{REFERENCES}

[1] Cunningham-Rundles C, Bodian C. Common variable immunodeficiency: clinical and immunological features of 248 patients. Clin Immunol 1999; 92: 34-48.

[2] Agematsu K, Futatani T, Hokibara S et al. Absence of memory B cells in patients with common variable immunodeficiency. Clin Immunol 2002; 103: 34-42.

[3] Bayry J, Hermine O, Webster DA, Levy Y, Kaveri SV. Common variable immunodeficiency: the immune system in chaos. Trends Mol Med 2005; 11: 370-6.

[4] Brouet JC, Chedeville A, Fermand JP, Royer B. Study of the B cell memory compartment in common variable immunodeficiency. Eur J Immunol 2000; 30: 2516-20.

[5] Wang JH, Avitahl N, Cariappa A et al. Aiolos regulates B cell activation and maturation to effector state. Immunity 1998; 9: 54353.

[6] Wang JH, Nichogiannopoulou A, Wu L et al. Selective defects in the development of the fetal and adult lymphoid system in mice with an Ikaros null mutation. Immunity 1996; 5: 537-49.

[7] Ghadiri A, Duhamel M, Fleischer A, Reimann A, Dessauge F, Rebollo A. Critical function of Ikaros in controlling Aiolos gene expression. FEBS Lett 2007; 581: 1605-16.

[8] Ayllon V, Fleischer A, Cayla X, Garcia A, Rebollo A. Segregation of Bad from lipid rafts is implicated in the induction of apoptosis. $\mathrm{J}$ Immunol 2002; 168: 3387-93.

[9] Romero F, Martinez AC, Camonis J, Rebollo A. Aiolos transcription factor controls cell death in $\mathrm{T}$ cells by regulating Bcl-2 expression and its cellular localization. EMBO J 1999; 18: 3419-30.

[10] Liippo J, Nera KP, Veistinen E et al. Both normal and leukemic B lymphocytes express multiple isoforms of the human Aiolos gene. Eur J Immunol 2001; 31: 3469-74.
[11] Duhamel M, Arrouss I, Merle-Beral H, Rebollo A. The Aiolos transcription factor is up-regulated in chronic lymphocytic leukemia. Blood 2008; 111: 3225-8

[12] Nakase K, Ishimaru F, Fujii K et al. Overexpression of novel short isoforms of Helios in a patient with T-cell acute lymphoblastic leukemia. Exp Hematol 2002; 30: 313-7.

[13] Caballero R, Setien F, Lopez-Serra L et al. Combinatorial effects of splice variants modulate function of Aiolos. J Cell Sci 2007; 120: 2619-30.

[14] Narvi E, Nera KP, Terho P, Mustonen L, Granberg J, Lassila O. Aiolos controls gene conversion and cell death in DT40 B cells. Scand J Immunol 2007; 65: 503-13.

[15] Boise LH, Gottschalk AR, Quintans J, Thompson CB. Bcl-2 and Bcl-2-related proteins in apoptosis regulation. Curr Top Microbiol Immunol 1995; 200: 107-21.

[16] Di Renzo M, Zhou Z, George I, Becker K, Cunningham-Rundles C. Enhanced apoptosis of T cells in common variable immunodeficiency (CVID): role of defective CD28 co-stimulation. Clin Exp Immunol 2000; 120: 503-11.

[17] Ferrer A, Ollila J, Tobin G et al. Different gene expression in immunoglobulin-mutated and immunoglobulin-unmutated forms of chronic lymphocytic leukemia. Cancer Genet Cytogenet 2004; 153: 69-72.

[18] Pokholok DK, Harbison CT, Levine S et al. Genome-wide map of nucleosome acetylation and methylation in yeast. Cell 2005; 122: 517-27.

[19] Nera KP, Lassila O. Pax5--a critical inhibitor of plasma cell fate. Scand J Immunol 2006; 64: 190-9.

[20] Morra M, Silander O, Calpe S et al. Alterations of the X-linked lymphoproliferative disease gene SH2D1A in common variable immunodeficiency syndrome. Blood 2001; 98: 1321-5.

(C) Billot et al.; Licensee Bentham Open.

This is an open access article licensed under the terms of the Creative Commons Attribution Non-Commercial License (http://creativecommons.org/licenses/by$\mathrm{nc} / 3.0 /$ ) which permits unrestricted, non-commercial use, distribution and reproduction in any medium, provided the work is properly cited. 